\title{
Perception of the Ponzo illusion by rhesus monkeys, chimpanzees, and humans: Similarity and difference in the three primate species
}

\author{
KAZUO FUJITA \\ Primate Research Institute, Kyoto University, Inuyama, Japan
}

\begin{abstract}
In Experiment 1, 3 rhesus monkeys and 1 chimpanzee were tested for their susceptibility to the Ponzo illusion. The subjects were first trained to report the length of the target bar presented at the center of the computer display by touching either of the two choice locations designated as "long" or "short." When inverted-V context lines were superimposed on the target bar, the subjects tended to report "long" more often as the apex of these upward-converging lines approached the target bar. The perception of the Ponzo illusion was thus demonstrated. In Experiment 2, the same 3 rhesus monkeys and 2 new chimpanzees were tested using two types of context lines that provided different strengths of linear perspective. The subjects showed a bias similar to that found in Experiment 1, but there was no difference in the magnitude of the bias between the two types of context in either species. This failed to support the classic account for the Ponzo illusion, the perspective theory, raised by Gregory (1963). In Experiment 3, the magnitude of the illusion was compared between the inverted- $V$ context and the context consisting of short vertical lines having the same gap as the former in the same 3 rhesus monkeys and 2 of the chimpanzees from the preceding experiments. While the chimpanzees showed the illusion for both types of stimuli, the monkeys showed no illusion for the latter. In Experiment 4, 6 humans were tested in a comparable procedure. As in the nonhuman primates, the illusion was unaffected by the strength of linear perspective. On the other hand, the humans showed considerably larger illusion for the context consisting of vertical lines than for contexts consisting of converging lines. Thus, there was a great species difference in the effect of the gap itself on the magnitude of the Ponzo illusion. Similarity found at first turned out to be no more than superficial. Possible sources of this species difference are discussed.
\end{abstract}

Nonhuman primates are commonly used as a model in the study of human information processing, especially in neuroscience. The rationale of this lies in that they are the species closest to humans and at the least their fundamental perceptual processes ought to be similar to those of humans.

Actually, for example, perception of some natural and geometric patterns has been shown to be similar not only among primates but also among species from different taxa (pigeons: Blough, 1985; rhesus monkeys: Sands, Lincoln, \& Wright, 1982; chimpanzees: Matsuzawa, 1990; Tomonaga \& Matsuzawa, 1992). Similarity has also been found in how short-term memory processes work in pigeons, monkeys, and humans (Jitsumori, Wright, \& Cook, 1988; Jitsumori, Wright, \& Shyan, 1989; Sands \& Wright, 1980; Wright, Santiago, Sands, Kendrick, \& Cook, 1985).

This study was supported by Grants-in-Aid for Scientific Research, Ministry of Education, Science, and Culture, Japan (Nos. 04831005 and 06610069 ). The author wishes to thank Tetsuro Matsuzawa of the Primate Research Institute, Kyoto University, for his help in conducting experiments with chimpanzees. Thanks are also due Sumiharu Nagumo of the Primate Research Institute for his technical support. Correspondence should be addressed to K. Fujita, Department of Psychology, Faculty of Letters, Kyoto University, Yoshida-honmachi, Sakyo, Kyoto, 606-01, Japan (e-mail: fujita@kupsy.kyoto-u.ac.jp).
My target here is perception of geometric illusions. Geometric illusions have captured much attention among psychologists (e.g., Coren \& Girgus, 1978; Frisby, 1979; Imai, 1984) because they are supposed to represent characteristics of human perception in a clear and magnified fashion. Thus, they undoubtedly provide a very useful means to discuss the similarity and the difference in visual information processing in humans and nonhumans. Knowledge about illusory perception of animals may not only endorse the rationale of the use of the animals as a model of humans in case similarity is found but may also have implications for the models of human illusory perception because they are expected to suggest what is fundamental and what is derived in human vision.

Unfortunately, though there have been several demonstrations of illusory perception by nonhuman species (e.g., Bayne \& Davis, 1983; Benhar \& Samuel, 1982; Dominguez, 1954; Harris, 1968), very few studies have examined the effects of the systematic parametric change. Most of them fail to provide data sufficient to discuss the homology and analogy of the illusory perception of different species.

Among them, a series of experiments by Fujita, Blough, and Blough $(1991,1993)$ investigated in some detail perception of the Ponzo illusion by pigeons (Columba livia). After demonstrating that pigeons clearly perceived two versions of the Ponzo illusion in two different procedures, 
Fujita et al. (1991) examined the effect of the linear perspective superimposed on the illusory figures. According to Gregory's (1963) classical perspective theory, the linear perspective perceived by the observer either consciously or unconsciously elicits "inappropriate" compensation based on the size constancy, which leads to perceptual magnification of the figures that appear farther. However, there was actually no effect of linear perspective to enhance the illusory perception by pigeons. This result was consistent with the results of some human studies (Fineman \& Carlson, 1973; Georgeson \& Blakemore, 1973; Humphrey \& Morgan, 1965; Newman \& Newman, 1974). Fujita et al. (1993) examined the effects of the inclination of the V-shaped context lines on pigeon's perception of the Ponzo illusion. The magnitude of the illusion changed as the inclination of the context lines increased, and there was no difference between upwardconverging lines and downward-converging lines. In humans, on the other hand, some studies have shown that upward-converging lines are more powerful than are lines having other orientations (Brislin, 1974; Fisher, 1968).

Given these data showing that the perception of the Ponzo illusion may or may not be homologous between pigeons and humans, it ought to be important to test nonhuman primates whose visual systems are much more similar to those of humans. In the present study, rhesus monkeys and chimpanzees were tested using the same procedure, and the data were compared with those from humans tested in virtually the same procedure.

\section{GENERAL METHOD}

\section{Subjects}

The subjects were 3 rhesus monkeys (Macaca mulatta) and 3 chimpanzees (Pan troglodytes). The rhesus subjects were a 6-yearold male (Gonta), a 5-year-old female (Jusco), and a 6-year-old female (Ryuko). The chimpanzee subjects were an 18 -year-old female (Ai), a 16-year-old female (Pendesa), and a 14-year-old female (Chloe). All of these subjects were young adults. ${ }^{1}$ The subjects received no restriction of food throughout the experimental period; however, occasionally, a mild deprivation of food was imposed on the monkey subjects when their performances deteriorated considerably. The use of these subjects adhered to the Guide for the care and use of laboratory primates (Primate Research Institute, Kyoto University, 1986). Two female and 4 male adult humans (Homo sapiens), between 22 and 41 years of age, also served as subjects. All human subjects had normal or corrected-to-normal visual acuity.

\footnotetext{
Apparatus

Apparatus for rhesus monkeys. The experimental box was a monkey cage, $70 \times 70 \times 70 \mathrm{~cm}$. A 14-in. CRT monitor $(\mathrm{KX}-14 \mathrm{HD}$, Sony) was installed on one wall of the box. A touch sensor (Hypertouch, Nissha Intersystems) was mounted on the monitor. A universal feeder (S-100, Sanso Electronics) could deliver pieces of food, a mixture of sweet potatoes and monkey chow, into a food cup at the bottom of one panel of the experimental box. An electronic doorbell installed behind the panel signaled the delivery of food. A houselight at the top of the box provided the illumination of the experimental box during the experimental session. A personal computer (PC286VE, Epson) controlled the equipment.
}

Apparatus for chimpanzees. The chimpanzees were tested in an experimental chamber, $150 \mathrm{~cm}$ wide, $150 \mathrm{~cm}$ deep, and $170 \mathrm{~cm}$ high. The other equipment was basically the same as that used for the rhesus monkeys. However, the CRT monitor was 21 in. (FHC3I WEX, Mitsubishi), and the computer was a PC386GS, Epson. The food rewards were apples, bananas, and raisins.

Apparatus for humans. The human subjects were tested using a mouse instead of a touch sensor. A 14-in. CRT monitor (PC-KD862S, $\mathrm{NEC}$ ) and a personal computer (PC386GS, Epson) were used. The humans received 1,000 Japanese yen after the test session.

\section{Stimuli}

Stimuli were black computer graphic patterns on a white background. The spatial resolution was $640 \times 400$ pixels. Each stimulus had one target bar and a variety of context patterns on both sides of the target bar. One hundred pixels subtended $44 \mathrm{~mm}$ on the 14-in. monitors and $60 \mathrm{~mm}$ on the 21 -in. monitor. The viewing distance was about $30 \mathrm{~cm}$ for the rhesus monkeys and about $50 \mathrm{~cm}$ for the chimpanzees and the humans. Viewed from this distance, the visual angles of 100 pixels were $8.41^{\prime}$ for the rhesus monkcys, $6.88^{\prime}$ for the chimpanzees, and $5.04^{\prime}$ for the humans.

\section{General Procedure}

Figure 1 shows a rough image of a trial. Trials started with a stimulus at the center of the CRT monitor after the intertrial interval (ITI). The length of the ITI was $5 \mathrm{sec}$ for the rhesus monkeys, $3 \mathrm{sec}$ for the chimpanzees, and $1 \mathrm{sec}$ for the humans. Several touches on this stimulus (five for the rhesus monkeys and three for the chimpanzees) or a single click of the mouse button on it (for the humans) produced two choice locations at both bottom corners of the monitor. ${ }^{2}$ The two choice locations were assigned to the reports of "long" or "short." The subjects were required to choose one of the two choice locations, depending on the length of the target bar. The assignment of the two choice locations was counterbalanced across subjects.

Testing procedure for nonhuman subjects. The length of the target bar was $40,48,56,64,72$, or 80 pixels for the nonhuman subjects. In only one test for one of the chimpanzee subjects, the bar was $50,54,58,62,66$, or 70 pixels because of too high accuracy. The first three lengths were "short" and the last three were "long." In training trials, a single response to the choice location corresponding to the length of the bar was reinforced by either food (primary reinforcer) accompanied by a 0.5 -sec doorbell sound or a doorbell sound only (conditional reinforcer). The probability of the
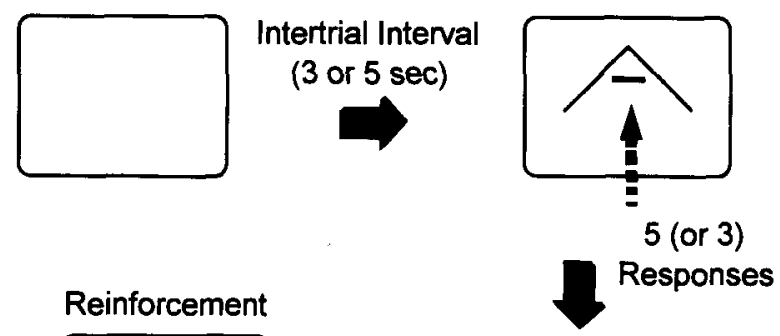

Reinforcement

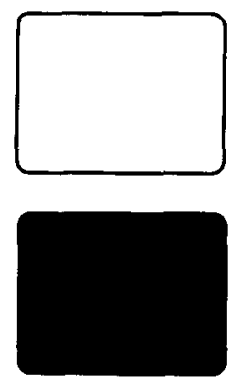

Correct

Timeout

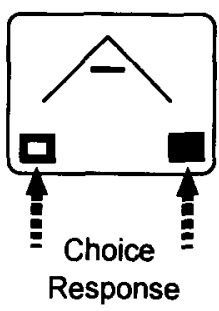

Figure 1. A schematic representation of the task. 
primary reinforcer was either $25 \%$ or $50 \%$ for the rhesus monkeys and either $50 \%$ or $100 \%$ for the chimpanzees. A single response to the other choice location was followed by a 5 -sec timeout, during which the CRT monitor went black. An ITI followed the reinforcement or the timeout.

All the subjects were first trained on the discrimination of the length of the horizontal bar presented without context patterns. In the first stage, the lengths of this target bar were 100 and 20 pixels. The target bar then was 80 and 40 pixels. After obtaining an accuracy of more than $90 \%$ correct for two consecutive sessions, all the six lengths listed above were presented. The number of trials per session was 384 . This training continued until the overall accuracy was more than $80 \%$ correct, the accuracies for the two extreme lengths were above $90 \%$ correct, and those for the two intermediate lengths were more than $50 \%$ correct for two consecutive sessions.

In each experiment, the subjects discriminated the length of the bar with a variety of context patterns superimposed on it. Test sessions were run after the criterion was reached. The test sessions consisted of 432 trials, 288 of which were baseline (training) trials and 144 were test trials. In the baseline trials, the subjects' responses were differentially reinforced as in training sessions. In the test trials, all the responses, either "long" or "short," were nondifferentially reinforced either primarily or conditionally (i.e., an all-reinforcedprobe procedure was followed). The stimuli that appeared in the test trials will be described later in each experiment. The test sessions were repeated 3-10 times, each separated by one or two training sessions. At the end of the series of the test sessions for each type of context, an original training session in which target bars appeared without context patterns was conducted to ensure that the subjects' performances were based on the length of the target bar.

Testing procedure for human subjects. The human subjects were tested for one session using a titration of the bar lengths. The subjects were instructed to choose one of the two choice locations, depending on the length of the target bar. The test session consisted of 407 trials. The first 32 trials were training trials in which a target bar of 59 pixels or 61 pixels appeared without context patterns. The remaining 375 trials were titration trials in which a variety of con- text patterns appeared. The stimuli and the procedure of the titration will be described in detail later in the Method section of Experiment 4.

\section{EXPERIMENT 1 A Demonstration of Perception of the Ponzo Illusion in Nonhuman Primates}

\section{Method}

Subjects. All 3 rhesus monkeys and 1 chimpanzee (Ai) served as subjects.

Stimuli. The context pattern consisted of two upward-converging lines as shown in Figure 2a. This is called the inverted-V context. Each line was 140 pixels in vertical length and 2 pixels in thickness. The lines inclined $45^{\circ}$ and touched each other at the top, making up the apex of $90^{\circ}$. There were three vertical locations of the context pattern: low-context, middle-context, and high-context patterns. In the middle-context patterns, the converging lines appeared such that the target bar was located in the center of the context. In low-context patterns, the converging lines appeared 20 pixels below the middle-context patterns, thus making the distance between the apex of the context and the target bar shorter. In the high-context patterns, the context appeared 20 pixels above the middle-context patterns.

Procedure. Only middle-context patterns appeared in the baseline sessions, in which 384 trials were conducted. Three test sessions separated by at least two or more baseline sessions followed this training. In the probe trials of the test sessions, the three locations of context - high, middle, and low-appeared randomly at the same frequency. During the test phase, 24 test trials were run for each combination of the three locations of contexts and the six lengths of the target bar.

\section{Results and Discussion}

Figure $3 \mathrm{a}$ shows the results for the monkey subjects, and Figure $3 b$ shows the results for the chimpanzee. The a
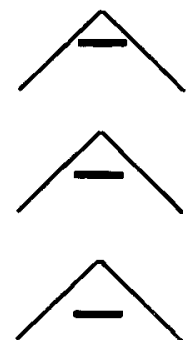

Inverted-V Context

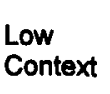

b

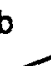

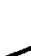
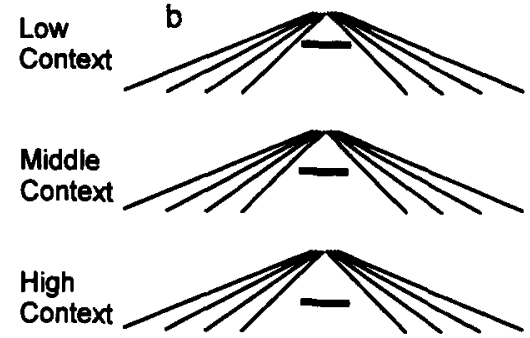

Perspective Context
C
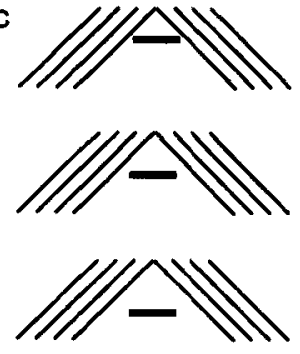

Non-Perspective

Context d

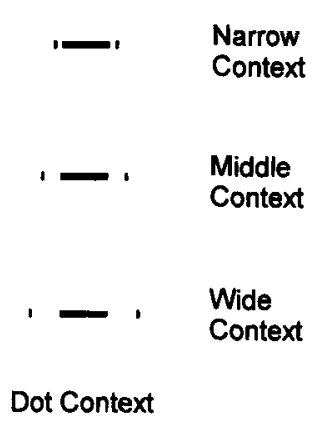

e
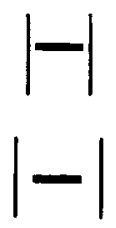

$1=1$

Vetical-Long-Line

Context

Figure 2. Examples of stimuli used in the present experiments. 
horizontal axis is the length of the target bar, and the vertical axis is the proportion of responses on the "long" key. The monkey data are the averages of the 3 subjects. A two-way analysis of variance (ANOVA) of bar length $X$ context location detected a significant main effect of the location of the context $[F(2,36)=4.50, p<.02]$ for the monkey data. Clearly, the subjects repoited "long" more often as the apex of the context lines approached the target bar. Though the chimpanzee data did not reach statistical significance $[F(2,10)=1.07$, n.s.], partly because $n=1$, the tendency was similar to the monkey data. The ordering of the proportion of the "long" reports was not perfect. But this is probably because of a positional bias due to the novelty of the test patterns in this subject. Note that any unexpected response bias could develop through accidental reinforcement of responses on all-reinforced test trials. These results demonstrated that nonhuman primates perceive the Ponzo illusion. When we compare the length of the target bar for which the proportion of "long" responses was $50 \%$, it is evident that the monkeys overestimated the bar length in the lowcontext condition by about $6 \%$, compared with that in the high-context condition. The same magnitude of the illusion was about $4 \%$ for the chimpanzee.

\section{EXPERIMENT 2 Effects of Linear Perspective}

Experiment 2 addressed the "perspective" theory most clearly stated by Gregory (1963). The essence of this theory is, as described earlier, that the linear perspective perceived by the observer either consciously or unconsciously elicits an "inappropriate" compensation due to the size constancy; hence, the figures that appear farther look larger than they actually are. In the case of the Ponzo illusion, the target bars appear longer as they approach the apex of the converging lines because these context lines provide a linear perspective. Actually, linear perspective superimposed on the Ponzo figure was shown to enhance the illusion in humans (Leibowitz, Brislin, Perlmutter, \& Hennessy, 1969). This theory appears to give qualitative support for some of other illusions as well.

\section{Method}

Subjects. All 3 rhesus monkeys and 2 chimpanzees (Pendesa and Chloe) served.

Stimuli. Two types of context - perspective context (Figure 2b) and nonperspective context (Figure 2c)-were prepared. Both types had four slanted lines on each side of the target bar. While these lines converged to one point in the former, they were parallel to each other and had no converging point in the latter. The innermost lines were exactly the same between the two types and matched those used in Experiment 1. As before, these contexts appeared in three different vertical locations: high, middle, and low.

Procedure. The subjects were trained using the middle-context condition of the two types of contexts. Ten test sessions separated by at least one or more baseline sessions followed this. In probe trials of the test sessions, all combinations of two context types, three vertical locations, and six bar lengths appeared randomly; thus, 40 test trials in total were run for each combination during the test phase.

\section{Results and Discussion}

Figure 4a shows the results for the monkey subjects, and Figure $4 \mathrm{~b}$ shows the results for the chimpanzee. The data are the averages of the subjects. The left column is for the perspective context, and the right column is for the nonperspective context. Again, the subjects reported "long" more often for the low-context condition than for the high-context condition. A three-way ANOVA of bar length $\times$ context location $\times$ context type suggested significant main effect of context location $[F(2,72)=11.42$, $p<.001$, for the monkeys; $F(2,36)=8.88, p<.002$, for the chimpanzees]. The ordering of the proportion of "long" reports was not perfect for low- and middle-context conditions of the perspective context in the chimpanzees. As in Experiment 1, this was probably due to a positional bias resulting from the novelty of the test patterns. It should be noted that ordering was perfect for the high- and lowcontext patterns, for which novelty was the same.

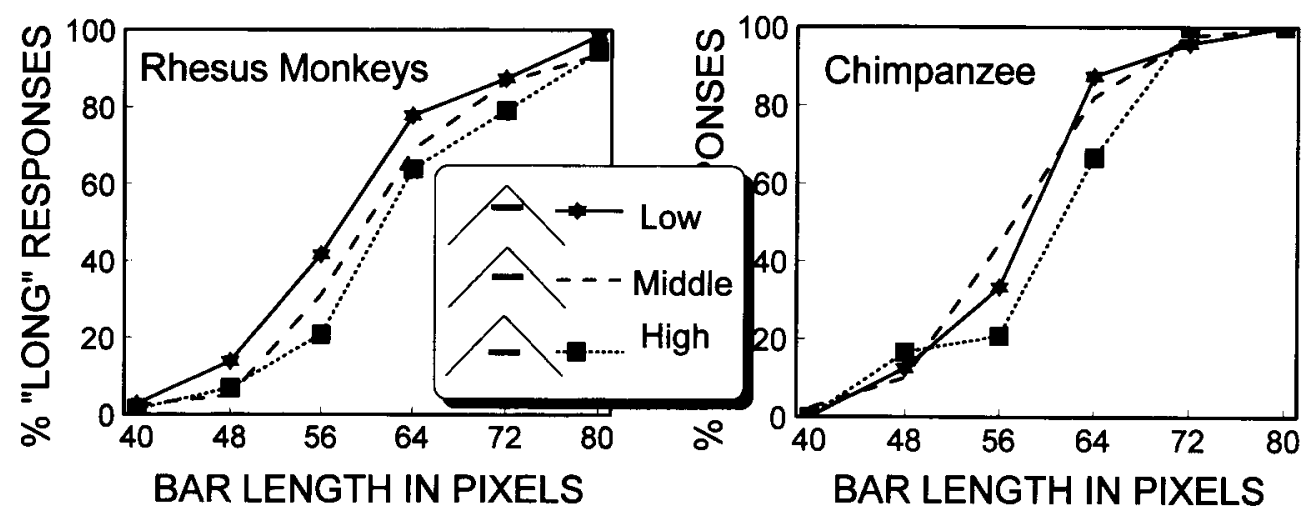

Figure 3. The results of Experiment 1. The vertical axis is the proportion of the subjects' "long" responses, and the horizontal axis is the length of the target bars in graphic pixels. (a) Average of the 3 rhesus monkeys. (b) Data from 1 chimpanzee. 
a

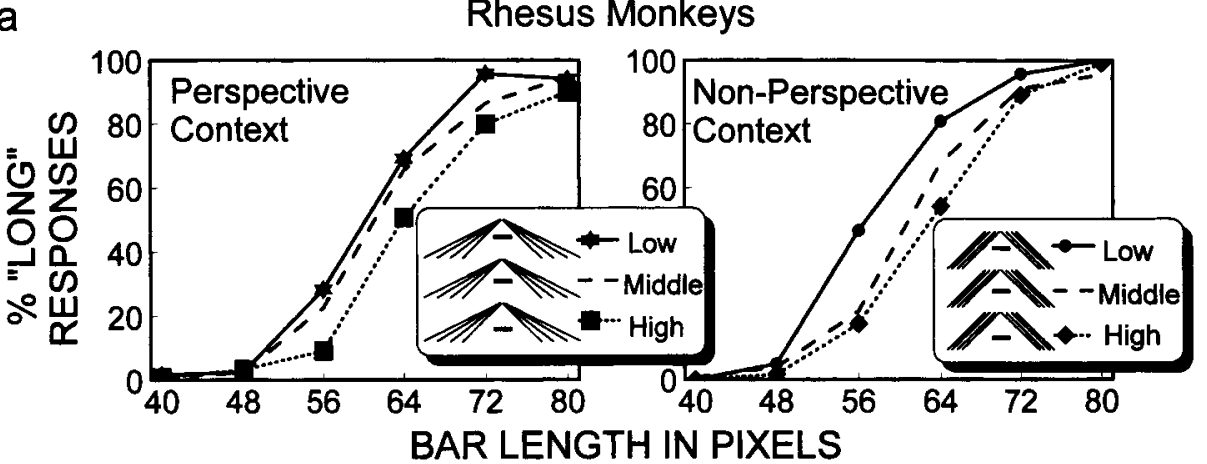

b

Chimpanzees

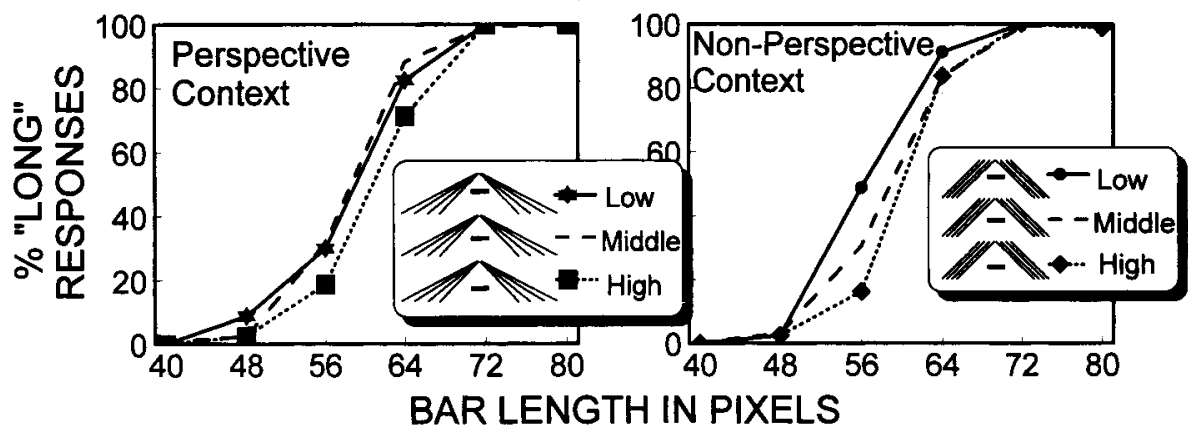

Figure 4. The results of Experiment 2. The left columns are for the perspective context and the right columns are for the nonperspective context. (a) Average of the 3 rhesus monkeys. (b) Average of the 2 chimpanzees.

On the other hand, there was no tendency that the illusion was stronger for the perspective context. Rather, the effect of the context location was apparently a little stronger for the nonperspective context than for the perspective context. But, statistically, there was no interaction between the context location and the context type $[F(2,72)=0.624$, n.s., for monkeys; $F(2,36)=1.25$, n.s., for chimpanzees]. The perspective theory received no support at least in this analysis in nonhuman primates.

\section{EXPERIMENT 3 \\ Effects of the Gap Between the Target Bar and the Context Patterns}

Fisher $(1969,1973)$ proposed that the proximity of the contours determines the Ponzo illusion. In human literature, this theory receives support by the fact that the amount of the illusion was virtually the same even when the four separate dots were placed in both sides of the two horizontal bars instead of the converging lines (Yamagami, 1978).

\section{Method}

Subjects. The same 3 rhesus monkeys and 2 chimpanzees (Ai and Pendesa) served.

Stimuli. Two types of context were used. One was the inverted$\mathrm{V}$ context used in Experiment 1 (Figure 2a). The other was the dot context, in which there were two separate short vertical lines that had the same gap between the context and the target bar as the
inverted-V context (Figure 2d). The thickness of the dot context was 2 pixels, and the vertical length was the same as the thickness of the target bar (i.e., 10 pixels).

The inverted- $V$ context appeared in the same three vertical locations as in Experiments 1 and 2. For the dot contexts, three different separations between the left dot and the right dot were prepared: narrow, middle, and wide context. The target-context gap of these three contexts was exactly the same as that of the low, middle, and high contexts, respectively.

Procedure. The subjects were trained with the middle contexts of both types of stimuli. Then, 10 test sessions were given, as was done in Experiment 2, for 2 of the rhesus monkeys and 1 chimpanzee (Ai). In total, 40 test trials were run for each combination of two context types, three locations (or widths), and six bar lengths. One of the rhesus monkeys (Ryuko) retired after the 6th test session because her performance suddenly dropped to a near-chance level. Thus, she received 24 trials for each stimulus combination. One of the chimpanzee subjects (Pendesa) received a more difficult discrimination than did the others, as noted in the General Method section (i.e., the step of the six bar lengths was half of those used for the other subjects). Also, she was tested only for 5 sessions, because her performance gradually increased too high even for this more difficult task. Thus, she received only 20 trials for each stimulus combination.

\section{Results and Discussion}

Figure 5a shows the results for the rhesus monkeys, and Figure $5 \mathrm{~b}$ shows the results for the chimpanzee subjects. The monkey data are the averages of the 3 subjects. Again, the illusion was demonstrated for the inverted-V context. The ordering of the proportion of "long" reports 
a

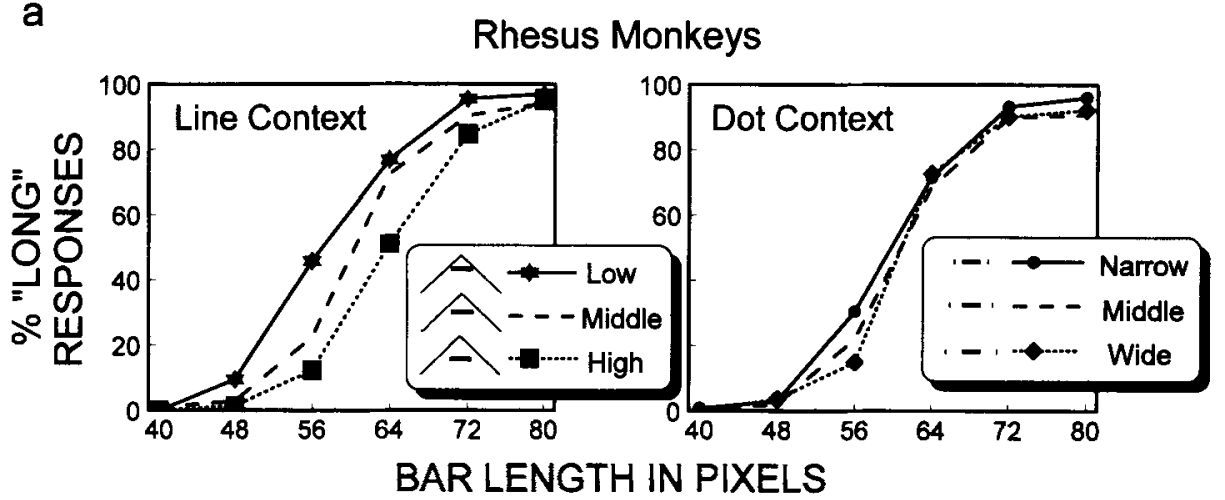

b

Chimpanzee (Ai)

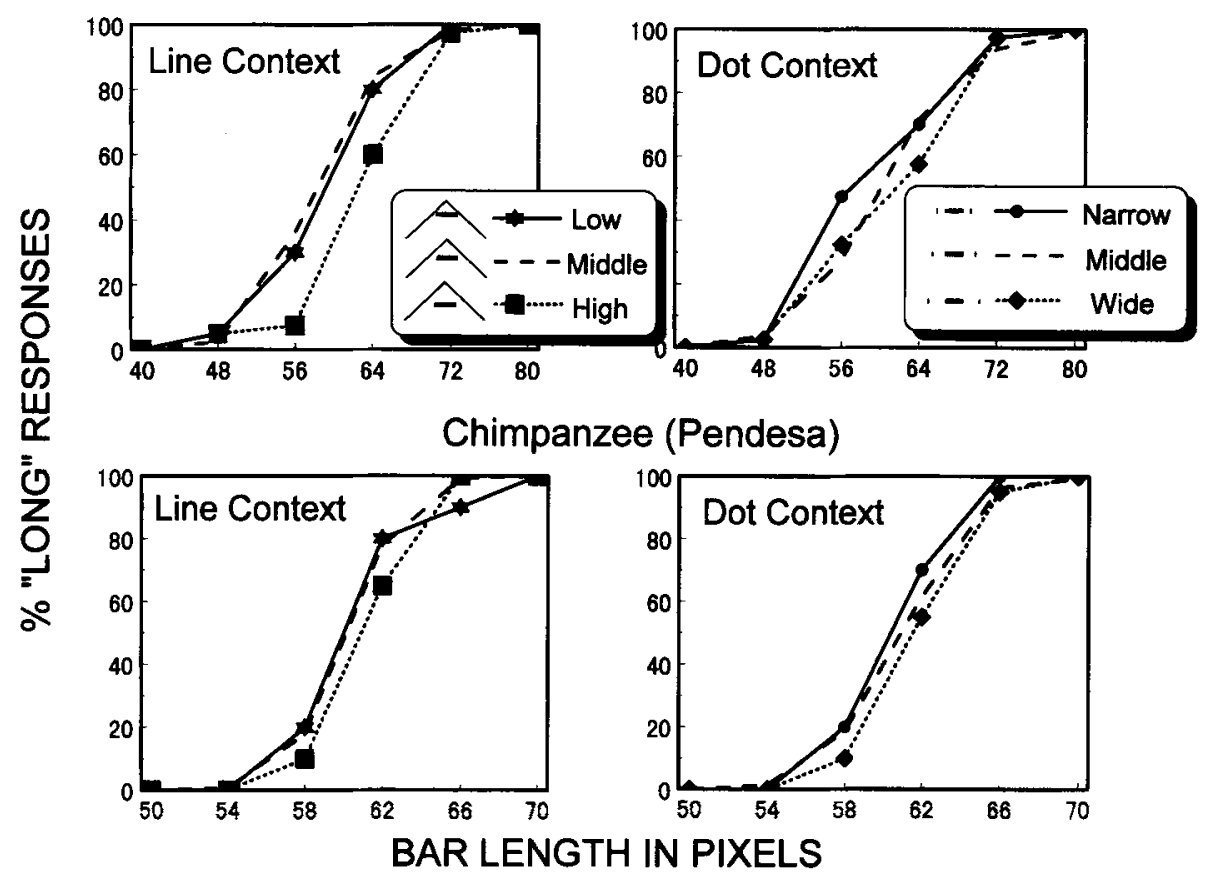

Figure 5. The results of Experiment 3. The left columns are for the inverted-V context, and the right columns are for the dot context. (a) Average of the 3 rhesus monkeys. (b) Data from the individual chimpanzees.

was not perfect for the three context locations in the chimpanzees, but, as before, low- and high-context conditions were almost perfectly ordered.

On the other hand, the effects of the dot context were different between the monkeys and the chimpanzees. In the chimpanzees, there was a bias similar to that shown for the inverted- $V$ context. However, in the rhesus monkeys, there was very little difference in the percent of "long" reports across the narrow, middle, and wide contexts. For the monkey data, the same three-way ANOVA as the one conducted in Experiment 2 now found both a significant main effect of context location (or width) $[F(2,72)=11.30, p<.001]$ and a significant interaction between the location (or width) of the context and the type of the context $[F(2,72)=3.80, p<.03]$. The data of the individual chimpanzees were separately analyzed by a three-way ANOVA with $n=1$. For both Ai and Pendesa, the main effect of context location (or width) was significant $[F(2,10)=4.64, p<.04$, for $\mathrm{Ai} ; F(2,10)=4.47$, $p<.05$, for Pendesa]. But, for both subjects, the interaction between context location (or width) and context type was not significant $[F(2,10)=1.58$, n.s., for Ai; $F(2,10)=0.33$, n.s., for Pendesa]. Even when the data of the 2 subjects were analyzed together, the interaction between context location (or width) and context type were not significant $[F(2,36)=0.54$, n.s.], whereas the main effect of context type was significant $[F(2,36)=6.06$, $p<.01]$. This suggests that the chimpanzee subjects perceived a similar illusion for the dot context, whereas the rhesus monkeys hardly did. In the monkeys, dot context 
was much less effective than was the inverted- $V$ context having the same gap size in inducing the illusion.

\section{EXPERIMENT 4 \\ Perception of the Ponzo Illusion by Humans}

\section{Method}

Subjects. All 6 human subjects served.

Stimuli. Stimuli were all five types of contexts listed in Figure 2. The vertical-long-line context had the same gap as the dot context. The vertical length was exactly the same as the inverted- $\mathrm{V}$ context. This was included because the monkeys' failure to show the illusion for dot context might have been due simply to weak contexts.

Procedure. As noted in the General Method section, the human subjects received one test session in which the length of the target bar was titrated. The test session consisted of initial 32 training trials and 375 test trials. During the training trials, the length of the target bar was either 59 pixels or 61 pixels. Correct choice responses simply advanced the trials, but incorrect responses were followed by a 1-sec timeout, during which the monitor went black. In the test trials that followed, 15 independent titration schedules ( 5 types of contexts $\times 3$ locations of contexts) ran simultaneously. The initial length of the target bar was 60 pixels. The titration followed a 2-up2-down Step 1 procedure-namely, the bar was shortened by 1 pixel when the subject reported "long" in 2 consecutive trials of the same stimulus patterns, and vice versa. The number of the titration trials was 25 per pattern.

\section{Results and Discussion}

Figure 6 shows the magnitude of the illusion in percent as a function of type of context. These values were calculated in the following way. First, the length of the target bar during the last 10 titration trials of each stimulus pattern was averaged. Suppose these values for one context pattern are $V_{\mathrm{H}}, V_{\mathrm{M}}$, and $V_{\mathrm{L}}$ for high, middle, and low context, respectively. The magnitude of the illusion for this context pattern is $\left(V_{\mathrm{H}}-V_{\mathrm{L}}\right) / V_{\mathrm{M}} \times 100$. In all types

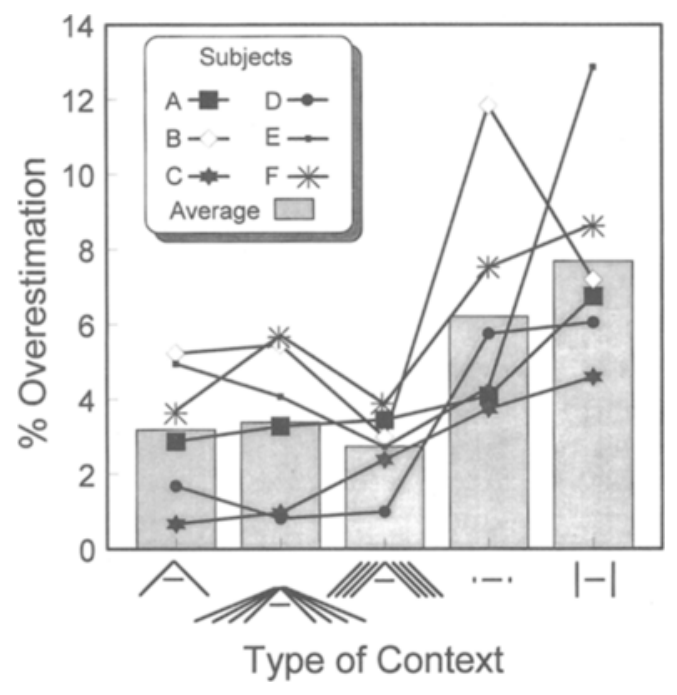

Figure 6. The results of Experiment 4 with human subjects. The vertical axis gives the magnitude of the illusion in percent; the horizontal axis, the type of context. Lines show data for individual subjects; the average appears in the histogram. of contexts, there was consistent ordering of these three values-namely, $V_{\mathrm{H}}>V_{\mathrm{M}}>V_{\mathrm{L}}$. The data for the individual subjects appear as line graphs, and the averages appear as histograms.

As is clear from the figure, the magnitude of the illusion did not differ for the three types of context consisting of converging lines (inverted-V, perspective, and nonperspective). This suggests that, even in humans, linear perspective superimposed on the Ponzo figure does not enhance the Ponzo illusion. This was consistent with the results for the rhesus monkeys and the chimpanzees.

On the other hand, the amount of the illusion was larger for the dot context and the vertical-long-line context. A one-way ANOVA using subject as block found a significant main effect of the context type $[F(4,20)=$ $9.11, p<.001]$. The Tukey pairwise comparison suggested a highly significant difference between vertical-long-line context and the first three contexts having converging lines $(p s=.002, .004$, and .001 , for the inverted-V, perspective, and nonperspective contexts, respectively) and a significant or nearly significant difference between dot context and the first three ( $p \mathrm{~s}=.054, .080$, and .022 , respectively). Actually, it is obvious that, for humans, it is quite easy to find that the illusion is clearer for dot and vertical-long-line contexts than for the others when we look at all the stimulus patterns shown in Figure 2. This was completely at odds with the data obtained for the rhesus monkeys. Though the data for the 2 chimpanzees in Experiment 3 are more similar to those of the humans, the amount of the illusion for dot context was comparable to that for inverted- $V$ context for the chimpanzees.

\section{GENERAL DISCUSSION}

From the results of the present experiments, it is now completely evident that at least two species of nonhuman primates perceive the Ponzo illusion. Both the chimpanzees and the rhesus monkeys consistently reported the length of the target bar to be long more often as it approached the apex of the converging lines.

Two of the potential sources of the Ponzo illusion were examined in this study. First, the effects of linear perspective superimposed on the figure were examined by comparing two types of contexts providing perspectives different in strength. No evidence was found that stronger linear perspective induces stronger illusion in any of the three primate species tested. The perspective theory proposed by Gregory (1963) thus received no support in primates, including humans. In testing pigeons using a similar procedure, Fujita et al. (1993) obtained similar results. These results may be more favorable to the account of this illusion based on the assimilation of the target bar to the context (e.g., Pressey, 1971; Pressey \& Epp, 1992). Animal's failure to be advantaged from linear perspective may be partly due to their difficulty to perceive perspective from line drawings (Cerella, 1977). However, this does not account for the human results. In addition, Newman and Newman (1974) demonstrated that Ponzo figures located in the patterns not suggesting depth were 
just as effective as those suggesting depth in inducing the illusion. Considering the reports suggesting positive effects of naturalistic pictures (Leibowitz et al., 1969) and correlation of the magnitude of this illusion with education, age, culture, and the capacity to perceive depth from two-dimensional drawings (Brislin, 1974; Brislin \& Keating, 1976; Kilbride \& Leibowitz, 1975; Predebon, 1984; Pressey, 1974; Quina \& Pollack, 1972; Smith, 1973; Wagner, 1977), it seems necessary to test the effects of more naturalistic pictures providing good perspective in nonhumans. Nonhuman primates have been shown to perceive natural pictures as a representation of real objects, and they at least discriminate social objects, such as individuals, species, or facial expressions (e.g., Dasser, 1987, 1988; Fujita, 1987, 1990, 1993a, 1993b; Fujita \& Watanabe, 1995; Kanazawa, 1996).

Second, effects of the size of the gap between the context and the target bar were examined. A very interesting species difference was found in this analysis: When the short vertical bars replaced the converging lines, the rhesus monkeys perceived virtually no illusion, whereas the humans perceived even stronger illusion. The chimpanzees, in this case, were somewhere between the humans and the rhesus monkeys. The source of this species difference ought to be addressed experimentally in the future. For now, a few potential accounts could be raised here. The first relates to the spatial anisotropy of the visual information processing. When organisms observe stimulus complex, there are interactions of each element figure. This is, of course, why we perceive the illusion at all. Also, there seems to be spatial anisotropy in the strength of these interactions. This explains why vertical Ponzo figures are more powerful in humans than are horizontal ones (Brislin, 1974). My point here is that there may be species differences in this spatial anisotropy. In monkeys, relative to in humans, stronger interaction might occur among vertically placed elements. If this is the case, portions of the figures above or below the target bar may have more power to induce illusion than may those on the right or left of the target bar, such as the dot context in the present study. Actually, in the monkeys, the magnitude of the illusion tended to change when the patterns at the top or bottom of all figures were modified with the patterns around the center constant (Fujita, 1994). As a related phenomenon, chimpanzees have been shown to have the greatest difficulty in recognizing horizontally located figures and faces rather than inverted ones (Fujita \& Matsuzawa, 1989; Tomonaga, Itakura, \& Matsuzawa, 1993). This hypothesis must be tested with all stimulus patterns from this study rotated by $90^{\circ}$.

The second hypothesis relates to the relative strength of assimilation and contrast. When inverted- $V$ patterns having only one target bar are presented, more assimilation effect comes from the part of the figures next to the target bar, whereas more contrast effect comes from the part of the context more distant from (i.e., above or below) the target. If monkeys are less susceptible to assimilation effect than to contrast effect, patterns having only short vertical bars next to the target may be less powerful in inducing the illusion.

It should be noted that there is a slight possibility that repeated use of the same nonhuman subjects in different experiments and difference in the size of the stimuli in terms of visual angle might have affected the obtained results. The effect of the former, however, seems minimal because the subjects' responses in the test trials were nondifferentially reinforced. The effect of the latter is not likely to be large, either, because the illusion was consistently found in all the species tested for the three stimulus patterns incorporating converging line context.

Whatever the reason might be, a most important suggestion of this species difference found here is that even fundamental visual information processing, such as perception of the length of the bar, could be greatly different among closely related species. This calls for caution against the careless and unlimited use of nonhuman primates as human models.

\section{REFERENCES}

Bayne, K. A. L., \& Davis, R. T. (1983). Susceptibility of rhesus monkeys (Macaca mulatta) to the Ponzo illusion. Bulletin of the Psychonomic Society, 21, 476-478.

Benhar, E., \& Samuel, D. (1982). Visual illusions in the baboon (Papio anubis). Animal Learning \& Behavior, 10, 115-118.

Blough, D. S. (1985). Discrimination of letters and random dot patterns by pigeons and humans. Journal of Experimental Psychology: Animal Behavior Processes, 11, 261-280.

Brislin, R. W. (1974). The Ponzo illusion: Additional cues, age, orientation, and culture. Journal of Cross Cultural Psychology, 5, 139-161.

Brislin, R. W., \& Keating, C. F. (1976). Cultural differences in the perception of a three-dimensional Ponzo illusion. Journal of Cross Cultural Psychology, 7, 397-412.

Cerella, J. (1977). Absence of perspective processing in the pigeons. Pattern Recognition, 9, 65-68.

COREN, S., \& GIRGus, J. S. (1978). Seeing is deceiving: The psychology of visual illusions. Hillsdale, $\mathrm{NJ}$ : Erlbaum.

DASSER, V. (1987). Slides of group members as representations of the real animals (Macaca fascicularis). Ethology, 76, 65-73.

DASSER, V. (1988). A social concept in Java monkeys. Animal Behaviour, 36, 225-230.

DomingueZ, K. E. (1954). A study of visual illusions in the monkey. Journal of Genetic Psychology, 85, 105-127.

Fineman, M. B., \& Carlson, J. (1973). A comparison of the Ponzo illusion with a textural analogue. Perception \& Psychophysics, 14, 31-33.

Fisher, G. H. (1968). Gradients of distortion seen in the context of the Ponzo illusion and other contours. Quarterly Journal of Experimental Psychology, 20, 212-217.

FISHER, G. H. (1969). Towards a new explanation for the geometrical illusions. I: The properties of contours which induce illusory distortion. British Journal of Psychology, 60, 179-185.

FISHER, G. H. (1973). Towards a new explanation for the geometrical illusions. II: Apparent depth or contour proximity? British Journal of Psychology, 64, 607-621.

FRISBY, J. P. (1979). Seeing: Illusion, brain, and mind. Oxford: Oxford University Press.

Fujita, K. (1987). Species recognition in five macaques. Primates, 28, 353-366.

FuJITA, K. (1990). Species preference by infant macaques with controlled social experience. International Journal of Primatology, 11, 553-573.

FUJITA, K. (1993a). Development of visual preference for closely related species by infant and juvenile macaques with restricted social experience. Primates, 34, 141-150. 
Fujita, K. (1993b). Role of some physical characteristics in species recognition by pigtail monkeys. Primates, 34, 133-140.

FujITA, K. (1994). Perception of the Ponzo illusion by rhesus monkeys and chimpanzees. (Research report of Grant-in-Aid for Scientific Research, Ministry of Education, Science, and Culture, Japan, No. 04831005, pp. 2-11). (in Japanese)

Fujita, K., Blough, D. S., \& Blough, P. M. (1991). Pigeons see the Ponzo illusion. Animal Learning \& Behavior, 19, 283-293.

Fujita, K., Blough, D. S., \& Blough, P. M. (1993). Effects of the inclination of context lines on perception of the Ponzo illusion by pigeons. Animal Learning \& Behavior, 21, 29-34.

Fujita, K., \& Matsuzawa, T. (1989). Comparison of the representational abilities of chimpanzees and humans: Short-term memory reproduction and mental rotation. Primate Research, 5, 58-74. (in Japanese with English summary)

FuiIta, K., \& WaTANABE, K. (1995). Visual preference for closely related species by Sulawesi macaques. American Journal of Primatology, 37, 253-261.

Georgeson, M. A., \& Blakemore, C. (1973). Apparent depth and the Mueller-Lyer illusion. Perception, 2, 225-234.

GREGORY, R. L. (1963). Distortion of visual space as inappropriate constancy scaling. Nature, 199, 678-680.

HaRRIS, A. V. (1968). Perception of the horizontal-vertical illusion by stumptail monkeys. Radford Review, 22, 61-72.

HOLt, G. L., \& SHAFER, J. N. (1973). Function of intertrial interval in matching-to-sample. Journal of the Experimental Analysis of Behavior, 19, 181-186.

Humphrey, N. K., \& Morgan, M. J. (1965). Constancy and the geometric illusions. Nature, 206, 744-745.

ImAI, S. (1984). Sakushi zukei: Miekata no shinrigaku [Figures of optical illusions]. Tokyo: Science-sha.

Jitsumori, M., Wright, A. A., \& CoOK, R. G. (1988). Long-term proactive interference and novelty enhancement effects in monkey list memory. Journal of Experimental Psychology: Animal Behavior Processes, 14, 146-154.

Jirsumori, M., Wright, A. A., \& ShYAN, M. R. (1989). Buildup and release from proactive interference in a rhesus monkey. Journal of Experimental Psychology: Animal Behavior Processes, 15, 329-337.

KanaZaWA, S. (1996). Recognition of facial expressions in a Japanese monkey (Macaca fuscata) and humans (Homo sapiens). Primates, 37, 25-38.

KILBRIDE, P. L., \& LeIBOwITZ, H. (1975). Factors affecting the magnitude of the Ponzo perspective illusion among the Baganda. Perception \& Psychophysics, 17, 543-548.

Leibowitz, H., Brislin, R., Perlmutter, L., \& Hennessy, R. (1969, November 28). Ponzo perspective illusions as a manifestation of space perception. Science, 166, 1174-1176.

Lydersen, T., Perkins, D., \& Chairez, H. (1977). Effects of fixedratio sample and choice response requirements upon oddity matching. Journal of the Experimental Analysis of Behavior, 27, 97-101.

MAtsuzaWa, T. (1990). Form perception and visual acuity in a chimpanzee. Folia Primatologica, 55, 24-32.

NELSON, K. R., \& Wasserman, E. A. (1978). Temporal factors influencing the pigeon's successive matching-to-sample performance: Sample duration, intertrial interval, and retention interval. Journal of the Experimental Analysis of Behavior, 30, 153-162.

Newman, C. V., \& Newman, B. M. (1974). The Ponzo illusion in pictures with and without suggested depth. American Journal of Psychology, 87, 511-516.

Predebon, J. (1984). Age trends in the Mueller-Lyer and Ponzo illusions. British Journal of Developmental Psychology, 3, 99-103.
Pressey, A. W. (1971). An extension of assimilation theory to illusions of size, area, and direction. Perception \& Psychophysics, 9, 172-176.

Pressey, A. W. (1974). Age changes in the Ponzo and filled-space illusions. Perception \& Psychophysics, 15, 315-319.

Pressey, A. W., \& EPP, D. (1992). Spatial attention in Ponzo-like patterns. Perception \& Psychophysics, 52, 211-221.

Primate Research Institute, Kyoto University (1986). Saru-rui no shiiku-kanri oyobi shiyo ni kansuru shishin [Guide for the care and use of laboratory primates] [Brochure]. Kyoto: Author.

Quina, K., \& Pollack, R. H. (1972). Effects of test line position and age on the magnitude of the Ponzo illusion. Perception \& Psychophysics, 12, 253-256.

SandS, S. F., LinColn, C. E., \& Wright, A. A. (1982). Pictorial similarity judgments and the organization of visual memory in the rhesus monkey. Journal of Experimental Psychology: General, 11 1, 369-389.

SANDS, S. F., \& WRIGHT, A. A. (1980). Serial probe recognition performance by a rhesus monkey and a human with 10 - and 20 -item lists. Journal of Experimental Psychology: Animal Behavior Processes, 6, 386-396.

SMITH, T. (1973). The susceptibility of Xhosa groups to a perspective illusion. Journal of Social Psychology, 90, 331-332.

Thomas, J. R. (1979). Matching-to-sample accuracy on fixed-ratio schedules. Journal of the Experimental Analysis of Behavior, 32, 183-189.

Tomonaga, M., ItakuRa, S., \& Matsuzawa, T. (1993). Superiority of conspecific faces and reduced inversion effect in face perception by a chimpanzee. Folia Primatologica, 61, 110-114.

Tomonaga, M., \& Matsuzawa, T. (1992). Perception of complex geometric figures in chimpanzees (Pan troglodytes) and humans (Homo sapiens): Analyses of visual similarity on the basis of choice reaction time. Journal of Comparative Psychology, 106, 43-52.

WaGner, D. A. (1977). Ontogeny of the Ponzo illusion: Effects of age, schooling, and environment. International Journal of Psychology, 12, 161-176.

WiLliams, B. A. (1971). Color alternation learning in the pigeon under fixed-ratio schedules of reinforcement. Journal of the Experimental Analysis of Behavior, 15, 129-140.

Wright, A. A., Santiago, H. C., Sands, S. F., Kendrick, D. F., \& CoOK, R. G. (1985, July 19). Memory processing of serial lists by pigeons, monkeys, and people. Science, 229, 287-289.

YaMAGami, A. (1978). Two kinds of apparent size distortion in the Ponzo illusion. Japanese Journal of Psychology, 49, 273-279. (in Japanese with English summary)

\section{NOTES}

1. A conventional translation of the ages of rhesus monkeys and chimpanzees to those of humans is to multiply by 4 in the former species and by 1.5 in the latter.

2. These different ITIs and ratios were employed to facilitate learning of the baseline task by the nonhuman subjects. Previous reports showed that long ITIs and large ratio requirements to, or long durations of, instructional discriminative stimuli facilitate animal learning (e.g., Holt \& Shafer, 1973; Lydersen, Perkins, \& Chairez, 1977; Nelson \& Wasserman, 1978; Thomas, 1979; Williams, 1971). Once the task was learned, the same values were kept in the test series so as not to unnecessarily confuse the subjects.

(Manuscript received September 6, 1995; revision accepted for publication April 4, 1996.) 